\title{
Light harvesting by a spherical silicon microcavity
}

\author{
M. Garín, ${ }^{a, *}$ R. Fenollosa, ${ }^{b}$ P. Ortega, ${ }^{a}$ and F. Meseguer $^{b}$ \\ ${ }^{a}$ Grup de recerca en Micro i Nanotecnologies, Departament d'Enginyeria Electrònica, \\ Universitat Politècnica de Catalunya, c/ Jordi Girona 1-3, Barcelona 08034, Spain \\ ${ }^{b}$ Instituto de Tecnología Química (CSIC - UPV), Universitat Politècnica de Valencia, \\ Av. Tarongers s/n 46022, Valencia, Spain \\ * moises.garin@upc.edu
}

\begin{abstract}
Silicon colloids are presented as efficient absorbers in the VIS-NIR region. The theory of resonant absorption by Mie modes in a single high-index sphere is reviewed and engineering rules established. The presented model predicts enhanced absorption in the crystalline silicon band-to-band absorption region, with absorption efficiencies exceeding one in the VIS and excellent NIR response. A maximum resonant absorption efficiency close to 4 can be obtained at the violet region $(425 \mathrm{~nm})$, and values above 0.25 are possible in the bandgap edge at wavelengths up to $1400 \mathrm{~nm}$. Silicon colloids are proposed as a promising cost-effective, silicon saving, sunlight harvesters with improved VIS and NIR response.
\end{abstract}

\section{INTRODUCTION}

Optical microresonators confine light both in time and space [1]; i.e. light can be stored within a resonant cavity for long times at well defined resonant frequencies. Most papers dealing with micro and nanocavities concern about optical properties in the transparent region of the cavity's material, the quality factor $Q$ being the most important parameter describing their optical excellence. The higher the resonance's $Q$ factor, the longer the dwell time of photons in the cavity. Typical applications of high- $Q$ optical resonators include filters [2], lasers [3], and single-photon sources [4], just to name a few. Other works, however, deal with leaky optical resonators and focus on their absorption properties where cavity matching for maximum absorption [5], and not high $Q$ values, is pursued. This is the common approach followed in the design of selective thermal emitters for thermophotovoltaic energy conversion [6], for instance. Absorption by nanoparticles, typically metallic, has also been attracting the researchers' interest during the last years $[7,8,9,10]$ since they can scatter and absorb more 
light than incident on it [11]. Furthermore, it has been recently shown that metallic/dielectric multilayer particles can reach even higher scattering and absorption levels, what has been termed as superscattering [12] and superabsorption [9,10] respectively.

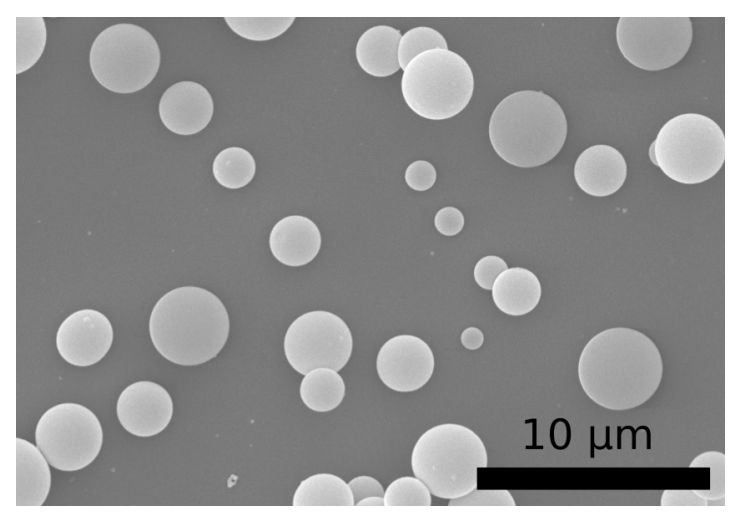

FIG. 1. SEM image, top view, of a bunch of silicon microspheres produced by CVD over a polished Si substrate.

A few years ago we reported over a novel kind of silicon-based material that we called silicon colloids $[13,14]$. These silicon particles (see Fig. 1), with typical dimensions ranging between $0.5-5 \mu \mathrm{m}$, exhibit a highly spherical and smooth shape, behaving as good optical resonators with very well defined Mie resonances. Other groups have also reported on the processing methods of silicon nano- and microcavities by chemical [15] and physical $[16,17,18,19,20]$ methods. One of the main features of these resonators, as opposed to conventional silica colloids, is the high refractive index of silicon, which confers unique properties to their Mie resonances. In particular, high-refractive index micro and nanoparticles exhibit very strong low order electric [21] and magnetic [17,22] resonances with high values of the scattering cross-sections, what enables a rich set of effects and applications in photonics [23], sensing [24], and specially as photonic couplers [25] and electronic devices [26] for solar energy harvesting.

A genuine advantage for silicon colloids stems from the fact that silicon is the most important semiconductor material in the electronic industry. Very recently we have shown experimentally [26] that a rectifying junction into a single silicon microsphere behaves as a photodiode with a strong response at the resonant frequencies. In fact, light trapped in the high-Q Mie resonances dwell in the microspheres for very long times, equivalent to traveling 
distances much larger than the typical thickness of a silicon wafer $(250 \mu \mathrm{m})$, therefore boosting IR absorption while photogenerated carriers can be quickly collected minimizing recombination losses. Our experimental devices worked as a solar cell and exhibited Mie features on the spectral response that extended in the IR at least up to $1600 \mathrm{~nm}$ due to the resonance-enhanced residual IR absorption of microcrystalline silicon. Millions of silicon microspheres could be handled in colloidal solutions and deposited in large surfaces for sunlight harvesting applications. Microspherical devices could be interconnected along a surface in a somewhat similar fashion to Sphelar's technology [27], or they could decorate the front side of a thin solar cell.

Several questions arise concerning light harvesting using silicon microspheres. Whether silicon micro- and nano-cavities can efficiently absorb VIS and IR radiation beyond the optical performance of standard Si technology, and how a practical design of such cell architecture would look like, are still open questions with tight links between them. In this work we are addressing the first question and report on the absorption properties of silicon colloids in the VIS-NIR region (400-1400 nm). In particular we determine the maximum resonant absorption efficiency, as a function of the wavelength, a single monocrystalline silicon sphere can achieve and the optimum particle size required for such enhancement. The structure of the paper is the following: first we will derive a set of engineering rules in order to find, in a single resonating mode, the $Q$-matching condition for maximum absorption as well as the maximum absorption efficiency expected. These rules will enable us to easily compute the maximum resonant absorption efficiency achievable at any frequency in the VISNIR range for crystalline silicon microspheres. Finally, we make an inroad into the potential of silicon colloids for sunlight harvesting, as compared to bulk silicon, in the context of photovoltaic application.

\section{ABSORPTION BY MIE RESONANCES.}

The interaction of light with a spherical particle is rigorously described by the LorenzMie solution of the Maxwell equations [28]. When a sphere is illuminated with a plane wave, light is extincted either by scattering or absorption. The total light absorbed and scattered by the particle can be accounted through equivalent absorption and scattering cross sections, $\sigma_{\mathrm{a}}$ and $\sigma_{\mathrm{s}}$, respectively; similarly, the total extinction cross section is $\sigma_{\mathrm{e}}=\sigma_{\mathrm{a}}+\sigma_{\mathrm{s}}$. It is convenient to define dimensionless efficiencies for absorption, scattering and extinction through 


$$
q_{a}=\frac{\sigma_{a}}{\pi r^{2}}, q_{s}=\frac{\sigma_{s}}{\pi r^{2}} \text { and } q_{e}=\frac{\sigma_{e}}{\pi r^{2}}
$$

where $r$ is the radius of the particle. Notice that all the above efficiencies can be greater than one. For a dielectric sphere embedded in air, the scattering efficiencies can be calculated as an infinite sum of multipolar contributions through [28]

$$
\begin{aligned}
& q_{e}=\frac{2}{x^{2}} \sum(2 m+1)\left(\Re\left(a_{m}+b_{m}\right\}\right), \\
& q_{s}=\frac{2}{x^{2}} \sum(2 m+1)\left(\left|a_{m}\right|^{2}+\left|b_{m}\right|^{2}\right),
\end{aligned}
$$

and

$$
q_{a}=q_{e}-q_{s},
$$

where $a_{\mathrm{m}}$ and $b_{\mathrm{m}}$ are respectively the electric and magnetic multipolar coefficients defined as

$$
a_{m}=\frac{\widetilde{n}^{2} j_{m}\left(x_{1}\right) \frac{d}{d x}\left[x j_{m}(x)\right]-j_{m}(x) \frac{d}{d x_{1}}\left[x_{1} j_{m}\left(x_{1}\right)\right]}{\widetilde{n}^{2} j_{m}\left(x_{1}\right) \frac{d}{d x}\left[x h_{m}^{(1)}(x)\right]-h_{m}^{(1)}(x) \frac{d}{d x_{1}}\left[x_{1} j_{m}\left(x_{1}\right)\right]}
$$

and

$$
b_{m}=\frac{j_{m}\left(x_{1}\right) \frac{d}{d x}\left[x j_{m}(x)\right]-j_{m}(x) \frac{d}{d x_{1}}\left[x_{1} j_{m}\left(x_{1}\right)\right]}{j_{m}\left(x_{1}\right) \frac{d}{d x}\left[x h_{m}^{(1)}(x)\right]-h_{m}^{(1)}(x) \frac{d}{d x_{1}}\left[x_{1} j_{m}\left(x_{1}\right)\right]}
$$

In these equations, $m$ is the mode number, $\tilde{n}=n+i k$ is the complex refractive index of the sphere, $x=2 \pi r / \lambda$ is the size parameter, $x_{1}=\tilde{n} x, r$ is the sphere radius, and $j_{\mathrm{m}}$ and $h_{\mathrm{m}}{ }^{(1)}$ are the spherical Bessel and spherical Hankel functions, respectively, of the first kind. The Mie spectra is, thus, an infinite sum of electric and magnetic modes, each one presenting multiple resonances. To refer to a single resonance we use the usual nomenclature $a_{\mathrm{m}, \mathrm{o}}$ and $b_{\mathrm{m}, \mathrm{o}}$, where $m=1,2,3, \ldots$ is the mode number, $o=1,2,3, \ldots$ is the order of the resonance and the letter, $a$ or $b$, represent an electric or magnetic multipolar mode respectively. The order count begins at the first resonance found as $x$ increases starting from zero. Through the text we will also refer to the individual contribution of a single mode $m$ to the extinction, scattering and absorption efficiencies, i.e. only considering one term of the summations in equations (2), (3) and (4). In these cases we use a prime notation $\left(q_{\mathrm{e}}^{\prime}, q_{\mathrm{s}}^{\prime}, q_{\mathrm{a}}^{\prime}\right)$ to differentiate them from the total efficiencies. 
Despite its simple geometry, the scattering and absorption spectra of spherical particles are rather complex, exhibiting plenty of high- $Q$ resonances which strongly depend on the refractive index. Furthermore, the scattering and absorption spectra of high-refractive-index particles, such as silicon microspheres, highly differ from those of low index particles, such as silica colloids, the former showing strong low order resonances with extremely high scattering efficiencies (See supplementary figure S1). In general, Mie scattering spectra in high-index materials can be described as a sum of Fano resonances [29, 30], i.e. individual Mie resonances exhibit an asymmetric line-shape; on the contrary the internal electric field, and therefore the absorption, of all Mie modes exhibit the classical Lorentzian line-shape [30]. In spite of this, the strongest resonances in the case of silicon, both in scattering and absorption, correspond to low order resonances no matter the mode number. These resonances also exhibit a nearly symmetric line-shape in scattering [30] (See supplementary figure S2 as an example). Consequently, and as far as we are concerned in calculating the maximum absorption of silicon spheres, we will consider Mie resonances as classical resonators with Lorentzian lineshapes in both absorption and scattering, which will greatly simplify the analysis. From here on, our concern will be to determine the right $n$ and $k$ values that maximize the absorption by a single given particle in a well defined Mie resonance. We will rely on elemental resonator theory in order to deduce easy rules and expressions to find the $Q$ matching condition that maximizes absorption. In particular, since we focus on silicon microspheres in the VIS-NIR region, we will assume a high refractive index material and a broad range of absorption levels.

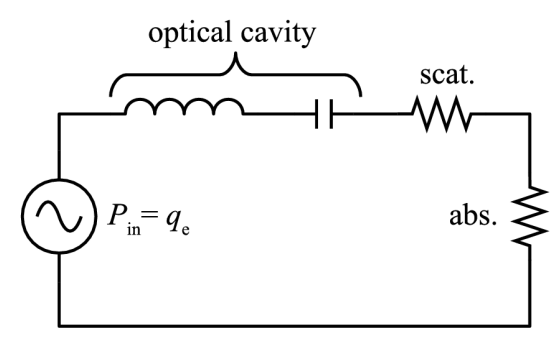

FIG. 2. Equivalent circuit

To any individual Mie mode, the general considerations of a simple resonator apply. The quality factor, $Q$, is determined by the cavity losses at the mode wavelength, namely scattering and absorption. Let us split $Q$ into the factors attributable to scattering, $Q_{\text {sca, }}$ and absorption, $Q_{\text {abs: }}$ : 


$$
Q^{-1}=Q_{s c a}^{-1}+Q_{a b s}^{-1}
$$

Here $Q_{\text {sca }}$ is associated to both, scattering due to the intrinsic spherical shape and due to local surface imperfections, while $Q_{\text {abs }}$ includes any absorption process such as semiconductor interband transitions (electron-hole generation) or free carrier absorption. For the sake of simplicity, if nothing else is stated, we will normally consider just the intrinsic spherical scattering and absorption due to electron-hole generation.

For low and moderate $k$ values $(k<<n)$, we can consider to a good approximation that $Q_{\text {sca }}$ is a function of the particle geometry and the refractive index but not of the extinction coefficient $k$ whereas $Q_{\text {abs }}$ will depend on both $n$ and $k$. The value of $Q_{\text {abs }}$ can be estimated from the average lifetime of photons free traveling in bulk medium before being absorbed. Following the Lambert's law, the mean distance, $L$, a photon travels is the reciprocal of the absorption coefficient $\alpha$. Considering a propagation speed $v=c / n$, the lifetime of photons can be approximated by

$$
\tau_{\mathrm{abs}}=\frac{L}{v}=\frac{n}{\alpha C}=\frac{n}{2 k \omega_{0}} .
$$

The absorption quality factor $Q_{\text {abs }}$ can, thus, be determined as

$$
Q_{\mathrm{abs}}=\tau_{a b s} \omega_{0}=\frac{n}{2 k} .
$$

The absorption in a particular sphere's resonance can be analyzed using a standard resonator analogy as the $R L C$ circuit shown in Fig. 2. In this analogy, the optical cavity would be represented by the capacitor and the inductor, while the scattering and absorption losses would be represented by the two respective resistors. The source serves as the incoming light and the power delivered by it represents the resonance's contribution to the total extincted light, $P_{\text {in }}=q^{\prime}$. Similarly, the power dissipated by the resistors would represent the contribution to the scattering and absorption efficiencies, $q_{\mathrm{s}}^{\prime}$ and $q_{\mathrm{a}}^{\prime}$, respectively. Notice that we are considering the contribution of a particular Mie resonance to the total scattering and absorption efficiencies, the total efficiencies being the sum of all modes' contributions. For low absorbing media, however, the total absorption efficiency in the vicinity of a resonance is defined only by the contribution of that particular resonance mode, i.e. $q_{\mathrm{a}}=q_{\mathrm{a}}^{\prime}$.

At a mode's resonant frequency, and using the above $R L C$ circuit as guide, it is easy to show that the extinction, scattering and absorption efficiencies scale with $Q$ as 


$$
\begin{aligned}
& q_{e}^{\prime} e^{\propto Q}=\frac{Q_{\mathrm{sca}} Q_{\mathrm{abs}}}{Q_{\mathrm{sca}}+Q_{a b s}}, \\
& q^{\prime}{ }_{s} \propto \frac{Q^{2}}{Q_{\text {sca }}}=\frac{Q_{\mathrm{abs}}^{2} Q_{\mathrm{sca}}}{\left(Q_{\mathrm{sca}}+Q_{a b s}\right)^{2}},
\end{aligned}
$$

and

$$
q_{a}^{\prime} \propto \frac{Q^{2}}{Q_{\mathrm{abs}}}=\frac{Q_{\mathrm{sca}}^{2} Q_{\mathrm{abs}}}{\left(Q_{\mathrm{sca}}+Q_{a b s}\right)^{2}} .
$$

As equation (12) shows, the maximum absorption efficiency $q_{\mathrm{a}}^{\prime}$ is achieved when the $Q$ matching condition $Q_{\mathrm{abs}}=Q_{\mathrm{sca}}$ is met. At this condition the total quality factor becomes $Q=Q_{\text {sca }} / 2$ which, in combination with Eq. (9), leads to

$$
k_{\text {max }}=\frac{n}{2 Q_{\text {sca }}}=\frac{n}{4 Q} .
$$

This expression is one of the main results of the paper and relates the real and imaginary parts of the refractive index ( $n$ and $k$ ) with the resonance's quality factor so that absorption is maximized. At the maximum absorption point $q_{\text {a }}^{\prime}$ and $q_{\mathrm{s}}^{\prime}$ also equilibrate and, by looking at Eq. (11), it can be deduced that they become equal to

$$
q_{a}^{\prime}\left(k=k_{\max }\right)=q_{s}^{\prime}\left(k=k_{\max }\right)=\frac{q_{s}^{\prime}(k=0)}{4} .
$$

In other words, the maximum absorption efficiency at a particular mode is $1 / 4$ of the contribution to the scattering efficiency of that same mode when considering no losses, just as it has been previously shown for nanoparticles in the vicinity of a surface plasmon [7]. Notice that Eq. 14 is only true for resonances with Lorentzian line-shape. Taking into account the above considerations, the effect of changing the refractive index $n$ becomes straightforward. Increasing $n$ leads toward an increase of the cavity confinement effect and, thus, toward an increase of the quality factor and the scattering efficiencies $q_{\mathrm{s}}$. Higher lossless $q_{\mathrm{s}}$ values imply higher $q_{\text {a }}$ values at the optimum $k$, as shown above. By the same rule, those particular modes that lead to the highest scattering efficiencies, like the lowest order ones in the case of silicon microspheres, will lead to the highest absorption efficiencies at ideal $Q$ matching conditions. We will further comment on this case on the light of the numeric results.

As an example, we show in Fig. 3 the evolution of $q_{\text {a }}^{\prime}$ (solid) and $q_{\text {s }}^{\prime}$ (dashed) as a function of $k$, obtained using the exact Mie solution, at three different modes, namely $b_{11,1}, b_{8,1}$ and $b_{5,1}$, and considering $n=3.42$ in all three cases. The $Q_{\text {sca }}$ factors $(k=0)$ for these modes are 
$8.7 \times 10^{7}, 5.4 \times 10^{5}, 3.9 \times 10^{3}$, respectively. Each resonance achieves optimum light absorption at a different $k$ values depending on $Q_{\text {sca }}$, peaking at roughly $1 / 4$ of the $q_{\text {s }}^{\prime}$ value for $k$ approaching zero, as expected. The optimum $k$ values given by Eq. (13) are $2.0 \times 10^{-8}, 3.2 \times 10^{-6}$ and $4.4 \times 10^{-}$ ${ }^{4}$, respectively, which are in perfectly good agreement with the exact peak positions seen in the figure. Dotted lines, shown for completeness, correspond to the total absorption efficiency $q_{\mathrm{a}}$, which is nearly identical to the individual resonance's contribution $q_{\mathrm{a}}^{\prime}$ except for very high $k$ values $\left(k>10^{-2}\right)$, where light is absorbed just by traveling a distance comparable to the particle size. For higher $k$ values, beyond the scope of this paper, $k$ becomes higher than $n$, behaving more like metal, and the absorption goes down again. For this reason, a maximum absorption appears for $k \approx 1$ which is independent of the frequency and that is not related to resonant phenomena. It is worth noticing in Fig. 3 that the maximum $q_{\mathrm{a}}$ values are higher for lower quality factor modes. This effect corresponds to the higher $q_{\mathrm{s}}$ values achieved for these particular modes, as mentioned earlier. This is a general trend of Mie modes in high-index materials. Low order modes, although with lower quality factors, tend to exhibit greater scattering efficiencies thanks to higher diffraction effects. As a result, the highest possible absorption efficiencies will be achievable with the fundamental resonances with lowest order and lowest mode number.

In summary, we have shown a simple relation between $n, k$ and $Q$ that allows to find the matching condition for maximum absorption efficiency. We have also shown that the maximum $q_{\mathrm{a}}$ is obtained when $q_{\mathrm{a}}$ and $q_{\mathrm{s}}$ equilibrate, and that it is $1 / 4$ of the $q_{\mathrm{s}}$ value when no absorption losses are considered. As a result, a high scattering efficiency, and not just a high $Q$ factor, is essential in order to reach high absorption efficiencies, which can be achieved with higher-index materials such as silicon. Since the presented analysis is based on a simple resonator, these relations are, in fact, universal and applicable to any dielectric-based optical resonator as far as the scattering of the analyzed modes can be approximated by a Lonrentzian line-shape. 


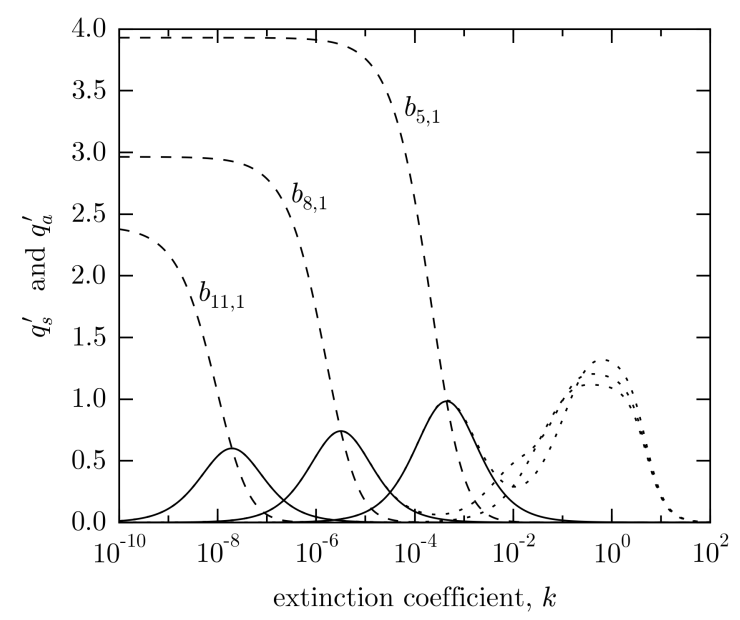

FIG. 3. Contribution to absorption (solid) and scattering (dashed) efficiencies vs extinction coefficient $k$ at selected mode wavelengths and constant refractive $n=3.42$. Selected modes are $b_{11,1}, b_{8,1}, b_{5,1}$; located at size parameters $4.37,3.38$, 2.36 and with quality factors $8.7 \times 10^{7}, 5.4 \times 10^{5}, 3.9 \times 10^{3}$, respectively. Solid and dashed curves represent the contribution of the modes to the scattering and absorption efficiencies respectively. Dotted curves represent the absolute particle absorption efficiency.

\section{VIS-NIR ABSORPTION BY SILICON COLLOIDS.}

In the previous section it has been presented the general absorption features of Mie resonances and the general relations that must be fulfilled in order to achieve maximum absorption at these resonant frequencies. Now we will particularize these results to a single silicon-based microsphere. Both real and imaginary parts of silicon's refractive index [31] have a strong dependence on wavelength around the visible band (see Fig. 4(a)). The real part ranges from 3.42 in the IR to nearly seven in the UV, while the imaginary part ranges from nearly zero in the near IR, up to more than five in the UV. Resonant behavior will be, thus, very different depending on the exact spectral region of interest. The typical size of the silicon colloids that we can produce lies between 0.5 and $5 \mu \mathrm{m}$, and smaller silicon particles can be produced by other techniques $[15,20]$. As a result, virtually any particular Mie mode, even the fundamental ones, can be located anywhere in the visible and NIR spectrum just by selecting the right particle size. The sphere diameter, thus, becomes an indirect way of tuning the complex refractive index for a particular Mie resonance. 
a
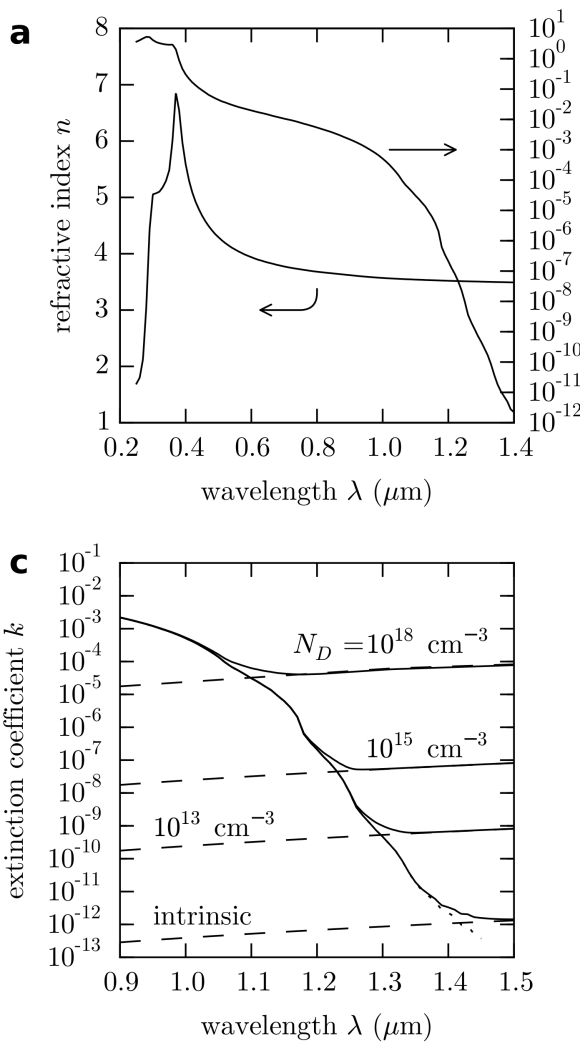
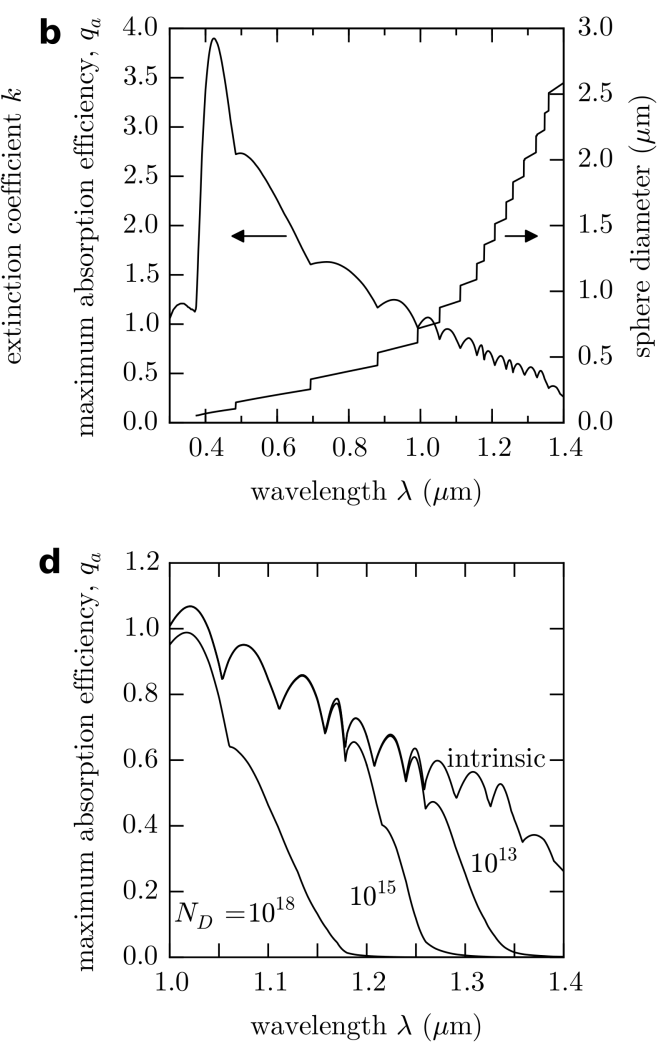

FIG. 4. (a) Optical properties of intrinsic crystalline silicon at room temperature. [31] (b) Maximum band-to-band absorption efficiency, and optimum spherical diameter, as a function of the wavelength for intrinsic silicon microspheres. (c) Silicon's extinction coefficient in the bandgap edge for different donor doping densities. Dashed lines correspond to the free-carrier contribution. (d) Maximum band-to-band absorption efficiency in the bandgap edge for a single silicon sphere with several doping densities.

Using common Mie computer routines [28], and with the aid of relation (13) and (14), we have determined the maximum absorption efficiency, $q_{\mathrm{a}}$, that can be achieved at each wavelength by choosing the appropriate particle dimension and considering intrinsic crystalline silicon. This is equivalent to say that, for each wavelength, we have considered the Mie resonance that absorbs the most given the $n$ and $k$ values at that particular point. Results are summarized in Fig. 4(b) for wavelengths up to $1400 \mathrm{~nm}$, which is the spectral region where silicon can directly convert photons to electrons. This curve represents the upper bound for the absorption efficiency of silicon particles. No absorption resonance in a silicon sphere can peak above this envelope curve. As can be seen in the figure, absorption efficiencies higher than one (antenna-like behavior) can be obtained throughout all the visible range, reaching nearly 4 in the violet region. This means that a single particle can absorb at that particular wavelength the equivalent light impinging on an equivalent area nearly 4 times its 
geometrical cross section. From a solar cell perspective, it is like if there was a concentrator built-in into the structure. Notice also that absorption efficiencies close to 1 can still be obtained at wavelengths larger than $1 \mu \mathrm{m}$, in the band-to-band absorption edge of silicon, where absorption is negligible.

Figure 4(b), shows an optimum case for every wavelength independently. This means that, for each wavelength, a mode fulfilling Eq. (13) has been selected. The actual $k$ value at every wavelength defines the $Q$ factor of the Mie mode that is best suited to enhance absorption at that particular wavelength. In the infrared, with very low $k$ values, high $Q$ factor modes are needed and, therefore, it results in bigger particle size values. In the ultraviolet region, with high $k$ values, low number modes are needed, resulting in smaller particle size values. The maximum $q_{\text {a }}$ curve shows different lobes, each one corresponding to a region dominated by the same resonance. When the resonance changes, a new lobe starts and the optimum diameter changes abruptly. All the calculated optimum resonances leading to Fig. 4(b) are first order $b$ modes, $b_{\mathrm{m}, 1}$, and the mode number $m$ increases sequentially as we move from short to longer wavelengths. Although slightly lower, $a$ modes lead to $q_{\text {a }}$ values close to those of $b$ modes. Notice that both the $Q$ factor of modes and silicon's $k$ value tend to increase with frequency; therefore, it is not possible to fulfill relation (13) in a wide spectrum band with a single sphere. A polydisperse constellation of spheres, however, could be used to increase absorption in the full band of interest. If the spheres are located close enough, scattering cross sections will overlap, optimizing volume filling and rising new multiple scattering effects that can help to enhance absorption in wider bands.

Beyond $1150 \mathrm{~nm}$, in the band-to-band absorption edge of silicon, the extinction coefficient is extremely small since two or more phonons are required in the process. Nevertheless, the appropriate high- $Q$ Mie resonances can exhibit remarkable peak absorption efficiencies. Even at $1400 \mathrm{~nm}, q_{\mathrm{a}}$ values close to 0.25 are predicted theoretically for optimum modes. At the band-gap edge, however, absorption due to free carriers also plays an important role, specially for doped material. The total silicon's absorption in this spectral region is the sum of the intrinsic band-to-band absorption plus the free carrier absorption: $k=k_{\mathrm{bb}}+k_{\mathrm{fc}}$. The resonance behavior will thus maximize both absorption processes and the total absorption efficiency attributable to each process will just be proportional to the extinction coefficient of each one. We have calculated the resonant band-to-band absorption at room temperature, taking into account free-carrier absorption, for different donor doping densities namely $N_{\mathrm{D}}=10^{13} \mathrm{~cm}^{-3}$, a light doping, $N_{\mathrm{D}}=10^{15} \mathrm{~cm}^{-3}$, a moderate doping, and $N_{\mathrm{D}}=10^{18} \mathrm{~cm}^{-3}$, a high 
doping density. We have used the parameterization of Strum and Reaves [32] for the freecarrier absorption calculation, leading to the extinction curves shown in Fig. 4(c). The resulting maximum band-to-band absorption efficiencies are shown in Fig. 4(d). As it can be observed, the calculated absorption efficiencies drop rapidly whenever the free-carrier absorption becomes dominant. For slight doping densities or intrinsic materials, strong absorption enhancements are still accessible in the near infrared in the case of monocrystalline silicon.

\section{PARTICULATE VS. BULK ABSORPTION.}

As it has been shown, silicon microspheres can exhibit very strong absorption efficiencies through the VIS-NIR region, especially at the resonant wavelength values. An ensemble of spheres polydisperse in size could, therefore, lead to absorption enhancements in wider spectral ranges and to substantial silicon saving in solar cell applications. A convenient way of comparing absorption by particulate and bulk media is through normalizing the absorption cross section to the particle volume, which is known as the volume absorption coefficient $\alpha_{\mathrm{v}}:[28]$

$$
\alpha_{v}=\frac{q_{a} s}{v}
$$

where $s=\pi r^{2}$ is the cross section of the particle and $v=(4 / 3) \pi r^{3}$ is the volume of the particle.

The volume absorption coefficient can be interpreted in terms of a volume complete filled with spheres of the same size (assuming no unavoidable air-gap) that retain their individual scattering/absorption efficiencies. Although simple, this coefficient can be directly compared, in a somewhat fair way, with the absorption coefficient $\alpha$ of bulk silicon by defining the absorption enhancement $\beta$ as [8]

$$
\beta_{\lambda}=\frac{\alpha_{v}}{\alpha}
$$

This enhancement is defined for a single wavelength $\lambda$, and $\beta>>1$ means that the particle is more efficient absorbing light than bulk material at that particular wavelength. In order to get a helpful insight on the microspheres' potential for wide band absorption we further define the following spectral averaged ratio, $\xi$, as a figure of merit: 


$$
\xi=\frac{\int_{\lambda_{1}}^{\lambda_{2}} \alpha_{v} E d \lambda}{\int_{\lambda_{1}}^{\lambda_{2}} \alpha E d \lambda} .
$$

This equation gives us information of how much better particulate silicon can be in front of bulk silicon in a defined spectral band $\lambda_{1}<\lambda<\lambda_{2}$, and for a particular irradiance spectra $E(\lambda)$. It is worth noticing that this figure of merit emphasizes the total power absorption enhancement by taking the $\alpha E$ product. Therefore, spectral regions with high $\beta$ values but low $E$ values will barely contribute to $\xi$. Figure 5 shows the figure of merit calculated as a function of the particle diameter and for different spectral ranges considering the normalized sun spectrum AM1.5G. Since we are interested in the band-to-band absorption, we will consider different spectral ranges between a certain wavelength $\lambda_{1}$ (indicated in the figure for each curve) and the bandgap edge, $\lambda_{2}=\lambda_{\mathrm{g}}=1400 \mathrm{~nm}$ as limited by the optical constants given in Fig $4 \mathrm{a}$. As the figure 5 shows, if we consider the full UV-VIS-NIR spectrum $(250 \mathrm{~nm}-1400 \mathrm{~nm})$, the particulate medium can only equal the bulk performance for an optimum particle size of $100 \mathrm{~nm}$. However, considering the VIS-NIR portion $(450 \mathrm{~nm}-1400 \mathrm{~nm})$, the idealized particulate media could outperform bulk media by a factor of five in the range of particle sizes between $100 \mathrm{~nm}$ and $200 \mathrm{~nm}$. In the NIR region $(750-1400 \mathrm{~nm})$, the theoretical improvement could reach 14 for an ideal particle size of $200 \mathrm{~nm}$, although noticeable improvements can be achieved for sizes up to $1 \mu \mathrm{m}$. At the same time, as we narrowed the spectral range the figure of merit shows increasing ripples with the sphere diameter, since it becomes more sensitive to a particular mode becoming optimum somewhere within this spectral range. For even narrower NIR spectral ranges (not shown in the figure) the maximum value of $\xi$ continues increasing monotonically.

The above result indicates that, as far as wide spectral bands are considered, silicon microspheres seem better suited for boosting absorption in the IR, even though the maximum peak absorption efficiencies can be achieved in the blue spectral region. On the other hand, thin and ultra-thin solar cells suffer from lower NIR absorption due to the low Si absorption volume. Considering an ultra-thin substrate of $10 \mu \mathrm{m}$, the absorption length of photons would become larger than $10 \mu \mathrm{m}$ already for wavelengths above $750 \mu \mathrm{m}$, which makes mandatory the use of advanced light trapping techniques in order to reach high efficiencies. As we have shown in Fig. 5, a polydisperse layer of Si spheres with diameters in the range 200-1000 nm would have the potential to boost VIS-NIR absorption while, at the same time, working as 
efficient light scatterers. This suggests that a hybrid structure, based on a thin film plus spheres, might be better suited for boosting absorption in the full spectrum. Figure 6 shows our vision of how such a solar cell might look like. This structure resembles previous designs incorporating low refractive index silica microspheres on top of a thin-film solar cell for lighttrapping purposes [33,34]. However, at variance with those former devices using low refractive index and low absorption dielectric particles, here the Si microspheres would play a double role: a passive role by scattering light into the thin layer and, at the same time, an active role by boosting VIS and NIR absorption. It is worth mentioning that, in addition to the absorption process of single spheres, multiple scattering phenomena of a random distribution (both in position and in size) of high refractive index nanoparticles would also appear $[35,36,37,38]$ giving rise to complex absorption spectra that would need to be maximized. This is, however, outside the scope of this paper, and it constitutes itself a field of research.

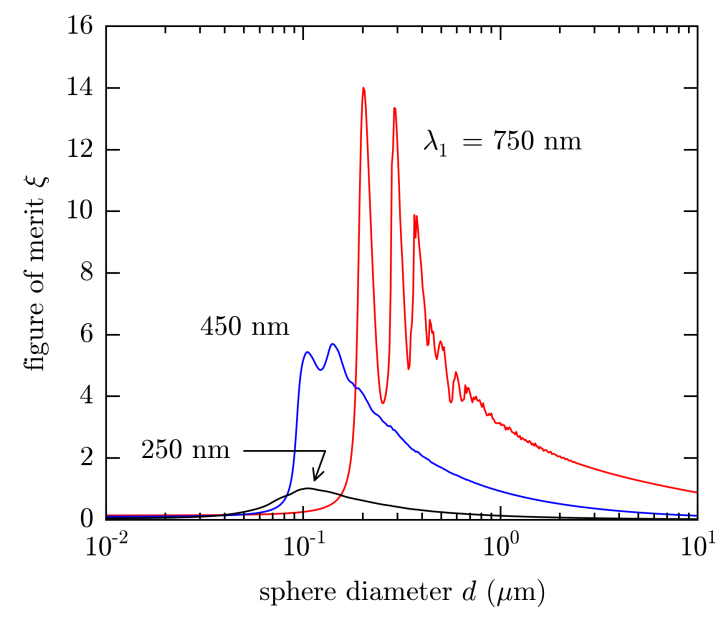

FIG. 5. Figure of merit for different wavelength bands $\lambda_{1}<\lambda<1400 \mathrm{~nm}$ as a function of the particles diameter. Only band-to-band absorption is considered.

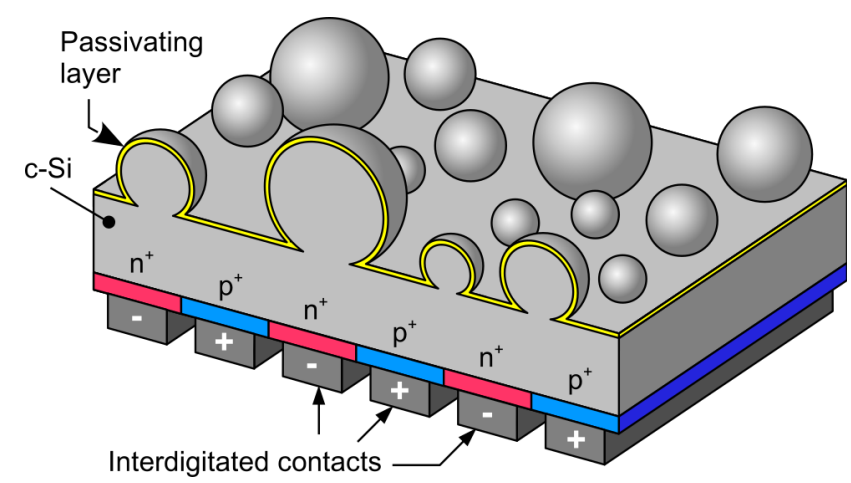

FIG. 6. Our view of an ultrathin solar cell incorporating silicon microspheres working as both scatterers and absorbers. 


\section{CONCLUSIONS}

We have presented silicon colloids as a powerful material for selective VIS-NIR detection with a high potential for cost-effective sunlight harvesting. We have explained the basic idea of absorption enhancement induced by Mie resonances and establishedthe general conditions to maximize it. It has been shown that silicon microspheres can exhibit resonant absorption efficiencies greater than one in the visible and efficiency values above 0.25 at wavelengths up to $1400 \mathrm{~nm}$, in the bandgap edge of Silicon. Finally, we have shown that silicon microspheres, compared to bulk Silicon, have potential to improve sunlight absorption in the whole NIR band, which could be especially beneficial in ultra-thin solar cell topologies.

\section{ACKNOWLEDGEMENTS}

The authors acknowledge financial support from the following projects: ENE201349984-EXP (Spanish Ministry of Economy and Competitiveness, MINECO), network 'Nanophotonics for Energy Efficiency' Grant Agreement 248855, MAT2012-35040 (MINECO), and PROMETEO/2010/043 Regional Government Project.

\section{REFERENCES}

${ }^{1}$ K. J. Vahala, Optical microcavities, Nature 424, 839-846 (2003).

${ }^{2}$ B. E. Little, S. T. Chu, H. A. Haus, J. Foresi, and J.-P. Laine, J. Lightwave Technol. 15, 9981005 (1997).

${ }^{3}$ S. L. McCall, A. F. J. Levi, R. E. Slusher, S. J. Pearton, and R. A. Logan, Appl. Phys. Lett. 60, 289-291 (1992).

${ }^{4}$ E. Moreau, I. Robert, J. M. Gérard, I. Abram, L. Manin et al., Appl. Phys. Lett. 79, 28652867 (2001).

${ }^{5}$ M. Ghebrebrhan, P. Bermel, Y. X. Yeng, I. Celanovic, M. Soljačić, and J. D. Joannopoulos, Phys. Rev. A 83, 033810 (2011).

${ }^{6}$ Y. X. Yeng, M. Ghebrebrhan, P. Bermel, W. R. Chan, J. D. Joannopoulos, M. Soljačić, and I. Celanovic, PNAS 109, 2280-2285 (2012).

${ }^{7}$ M. I. Tribelsky, EPL 94, 14004 (2011).

${ }^{8}$ B. S. Luk'yanchuk, A. E. Miroshnichenko, M. I. Tribelsky, Y. S. Kivshar, and A. R. Khokhlov, New J. Phys. 14, 093022 (2012).

${ }^{9}$ K. Ladutenko, P. Belov, O. Peña-Rodriguez, A. Mirzaei, A. E. Miroshnichenko, and I. V. Shadrivov, Nanoscale 7, 18897 (2015). 
${ }^{10}$ A. Mirzaei, I. V. Shadrivov, A. E. Miroshnichenko, and Y. S. Kivishar, Nanoscale 7, 17658 (2015).

${ }^{11}$ C. F. Bohren, Am. J. Phys. 51, 323 (1983).

${ }^{12}$ Z. Ruan, and S. Fan, Phys. Rev. Lett. 105, 013901 (2010).

${ }^{13}$ R. Fenollosa, F. Meseguer, and M. Tymczenko, Adv. Mater. 20, 95-98 (2008).

${ }^{14}$ R. Fenollosa, F. Ramiro-Manzano, M. Tymczenko, and F. Meseguer, J. Mater. Chem. 20, 5210-5214 (2010).

${ }^{15}$ J. T. Harris, J. L. Hueso, and B. A. Korgel, Chem. Mater. 22, 6378-6383 (2010).

${ }^{16}$ A. B. Evlyukhin, S. M. Novikov, U. Zywletz, R. L. Eriksen, C. Reinhardt, S. I. Bozhevolnyl, and B. N. Chichkov, Nano Letters 12, 3749-3755 (2012).

${ }^{17}$ A. I. Kuznetsov, A. E. Miroshnichenko, Y. H. Fu, J. Zhang, and B. Luk'yanchuk, Scientific Reports 2, 492 (2012).

${ }^{18}$ J.M. Geffrin, B. García-Cámara, R. Gómez-Medina, P. Albella, L.S. Froufe-Pérez, C. Eyraud, A. Litman, R. Vaillon, F. González, M. Nieto-Vesperinas, J.J. Sáenz, and F. Moreno, Nat. Commun. 3, 1171 (2012).

${ }^{19}$ A. Gumennik, L. Wei, G. Lestoquoy, A. M. Stolyarov, X. Jia, P. H. Rekemeyer, M. J. Smith, X. Liang, B. J.-B. Grena, S. G. Johnson, S. Gradečak, A. F. Abouraddy, J. D. Joannopoulos and Y. Fink, Nat. Commun. 4, 2216 (2013).

${ }^{20}$ U. Zywietz, A. B. Evlyukhin, C. Reinhardt, and B. N. Chichkov, Nat. Commun. 5, 3402 (2014).

${ }^{21}$ E. Xifré-Pérez, R. Fenollosa, and F. Meseguer, Optics Express 19, 3455-3458 (2011).

${ }^{22}$ L. Shi, T. U. Tuzer, R. Fenollosa, and F. Meseguer, Adv. Mater. 20, 5934-5938 (2012).

${ }^{23}$ E. Xifré-Pérez, J. D. Domenech, R. Fenollosa, P. Muñoz, J. Capmany, and F. Meseguer, Optics Express 19, 3185-3192 (2011).

${ }^{24}$ F. Ramiro-Manzano, R. Fenollosa, E. Xifré-Pérez, M. Garín, and F. Meseguer, Adv. Mater. 23, 3022-3025 (2011).

${ }^{25}$ Lei Shi, J. T. Harris, R. Fenollosa, I. Rodriguez, X. Lu, B. A. Korgel, and F. Meseguer, Nat. Commun. 4, 1904-1907 (2013).

${ }^{26}$ M. Garín, R. Fenollosa, R. Alcubilla, L. Shi, L.F. Marsal, and F. Meseguer, Nat. Commun. 5, 3440 (2014).

${ }^{27}$ K. Taira and J. Nakata, Nature Photonics 4, 602-603 (2010). 
${ }^{28}$ C. F. Bohren and D. R. Huffman, Absorption and Scattering of Light by Small Particles (Wiley-VCH, 2004).

${ }^{29}$ M. V. Rybin, K. B. Samusev, I. S. Sinev, G. Semouchkin, E. Semouchkina, Y. S. Kivshar, and M. F. Limonov, Optics Express 21, 30107-30113 (2013).

${ }^{30}$ M. I. Tribelsky, and A. E. Miroshnichenko, arXiv:1511.02931 [physics.optics] (2015).

${ }^{31}$ M. A. Green and M. J. Keevers, Prog. Photovolt: Res. Appl. 3, 189-192 (1995).

${ }^{32}$ J. C. Sturm and C. M. Reaves, IEEE Transactions on Electron Devices 39, 81-88 (1992).

${ }^{33}$ J. Grandidier, M. G. Deceglie, D. M. Callahan, and H. A. Atwater, Proc. of SPIE 8256, 825603 (2012).

${ }^{34}$ J. Grandidier, R. A. Weitekamp, M. G. Deceglie, D. M. Callahan, C. Battaglia, C. R. Bukowsky, C. Ballif, R. H. Grubbs, and H. A. Atwater, Phys. Status Solidi A 210, 255-260 (2013).

${ }^{35}$ A. Z. Genack, Phys. Rev. Lett. 58, 2043-2046 (1987).

${ }^{36}$ D. Wiersma, P. Bartolini, A. Lagendijk, and R. Righini, Nature 390, 671-673 (1997).

${ }^{37}$ A. A. Chabanov and A. Z. Genack, Phys. Rev. Lett. 87, 153901 (2001).

${ }^{38}$ S. Faez and Ad Lagendijk, Phys. Rev. Lett. 103, 053903 (2009). 


\section{Supplementary material.}

Influence of the refractive index $\boldsymbol{n}$ on the scattering and absorption efficiencies in a dielectric sphere.

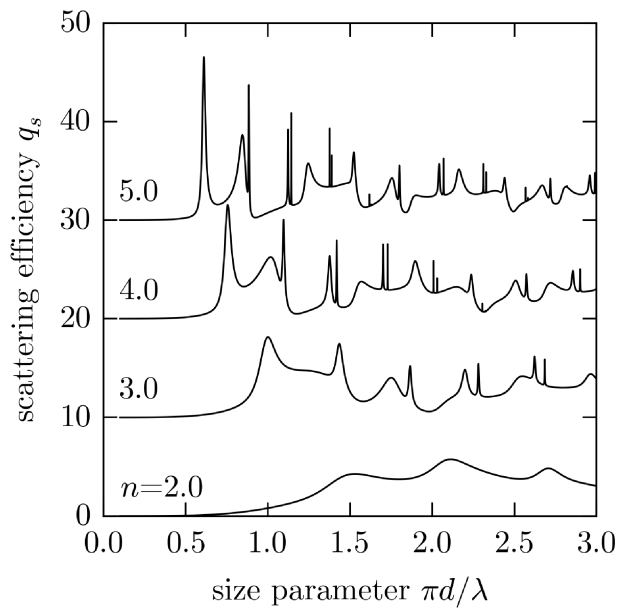

a

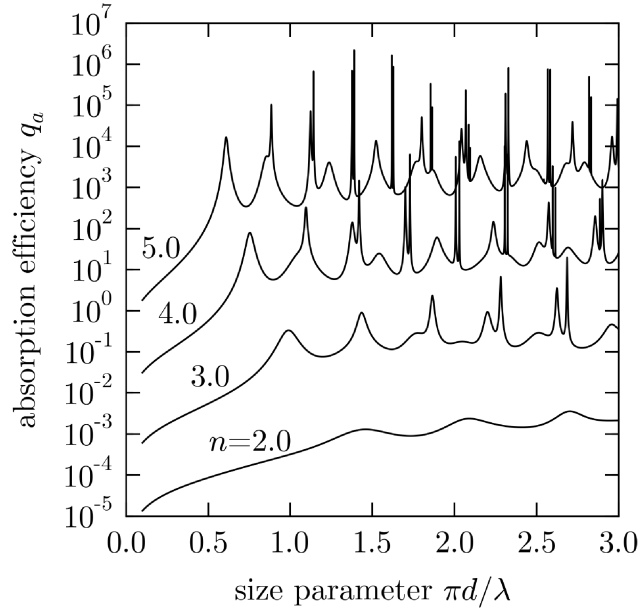

b

Fig. S1. (a) Scattering efficiency spectra $q_{s}$ for particles of different refractive index $n$. Curves are shifted by 10 for clarity. (b) Absorption efficiency $q_{a}$ as a function of the refractive index $n$. Curves are shifted two decades for clarity. In both figures, all curves where calculated for a constant extinction coefficient $k=10^{-}$ 4 .

\section{Lineshape of modes: scattering vs absorption.}

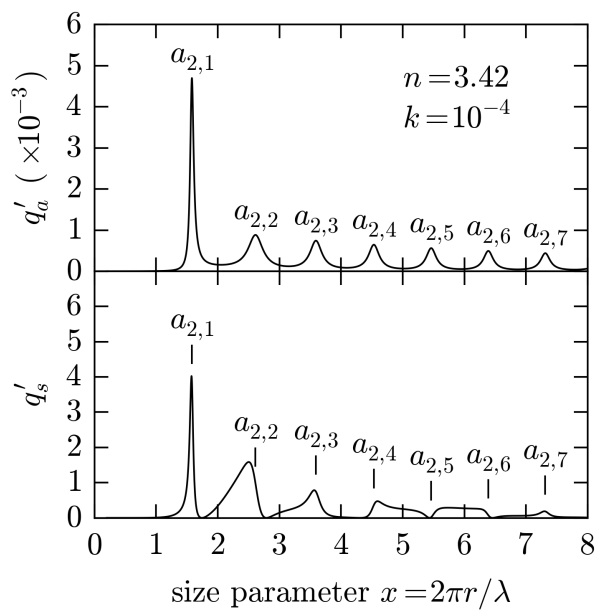

Fig. S2. Contribution to the scattering $q_{s}^{\prime}$ and to the absorption $q_{a}^{\prime}$ spectra of the mode $a_{2}$. In scattering, the vertical dashes indicates the exact resonance position. Calculations were done for a material with $n=3.42$ and $k=10^{-4}$. All modes exhibit a Lorentzian line-shape in absorption while they show a Fano line-shape in scattering. Notice that the first order mode $\left(a_{2,1}\right)$, however, also shows a line-shape close to a classical Lorentzian. 